\title{
Comparison of Intranasal Midazolam and Rectal Diazepam as Anticonvulsant in Children
}

\author{
Gunawan $\mathrm{PI}^{1}$, Rulian $\mathrm{F}^{2}$, Saharso $\mathrm{D}^{3}$
}

\begin{abstract}
Introduction: Rectal diazepam is reputed as the gold-standard management of childhood seizures. Otherwise, intranasal (IN) midazolam has no first-pass metabolism and faster onset of action. The effectiveness and easier route of these drugs are important choices for faster seizure cessation. The aim of this study was to clarify the effectiveness of intranasal midazolam compared with rectal diazepam for seizure termination. Material and Methods: The children, one month until 18 years of age, presented with acute seizures. Patients were randomly classified into two groups with either received intranasal midazolam or rectal diazepam for seizure termination. Interval time of drug administration to cease seizure was compared. The log-rank analysis was used for statistical analysis. Side effect of both drugs were evaluated. Results: There were 60 patients enrolled the study, 30 in each group. The median time interval for seizures cessation with intranasal midazolam was 42 seconds, otherwise in rectal diazepam group was 180 seconds. There was statistically significant difference interval time between two groups $(p<0.01)$. None of the both groups had any significant side effects statistically. Conclusion: Intranasal midazolam is effective to terminate a seizure in children. It can be used as an alternative treatment for seizures in patients with intravenous or rectal route difficulties.
\end{abstract}

Key words: Seizures, Intranasal midazolam, Rectal diazepam, Children, Effective

\section{Introduction}

Cizures are the well-known most common pediatric neurologic disorder, with $4 \%$ to $10 \%$ of children will experience minimum one seizure in the first 16 years of life ${ }^{1}$. Most childhood convulsions are short duration and resolve without treatment. Recommendations for early use of effective medication that could reduce seizure duration has been supporting to decrease morbidity and mortality ${ }^{2}$. Most patients with forcefull seizures will require pharmacologic medication to terminate the symptoms. Benzodiazepines are the recommended chosen drugs for the acute management of seizures. Diazepam is a benzodiazepine that has short duration of action and a rapid onset of action. Rectal diazepam is reputed as the goldstandard of prehospital medication of acute childhood convulsions.
'Dr. Prastiya Indra Gunawan, ${ }^{2} \mathrm{Dr}$. Fadil Rulian, ${ }^{3} \mathrm{Dr}$. Darto Saharso. All from the Paediatric Neurology, Airlangga University Surabaya, Indonesia

\author{
Address for correspondence: \\ Dr. Prastiya Indra Gunawan \\ Paediatric Neurology \\ Airlangga University \\ Surabaya, Indonesia \\ E-mail: prastiya_ig@yahoo.co.id \\ Tel: + 628113429476
}

\section{How to cite}

Gunawan PI, Rulian F, Saharso D. Comparison of Intranasal Midazolam and Rectal Diazepam as Anticonvulsant in Children. J Nepal Paediatr Soc 2015;35(2):117-122.

doi: http://dx.doi.org/10.3126/jnps.v35i2.13581

This work is licensed under a Creative Commons Attribution 3.0 License.

\section{(c) (1)}

Because diazepam can accumulates in fat stores, repeated doses can precede to a prolonged stage of sedation. Hypotension and cardio-respiratory depression may also occur. Administration of rectal diazepam might be embarrassing, and absorption is variable. Rectal diazepam has some disadvantages, slow drug absorption, duration and relatively short half-life, the drug often comes out with feces ${ }^{3,4}$. The use of intravenous (IV) access is difficult, thus requiring more time ${ }^{3,5,6}$.

Midazolam is imidazobenzodiazepine with sedative, muscle relaxant, anxiolytic, amnesic, and antiseizure properties. It is more powerful than diazepam. It could 
be administered intramuscularly, intravenously, intranasally as well buccally. Intranasal Midazolam route can go through the blood-brain barrier and reach the central nervous system biophase quickly ${ }^{7,8}$. Wermeling found some of the advantages of IN; no first-pass metabolism, more rapid absorption, faster onset of action, good bioavailability ${ }^{9}$. It is short-acting, and at physiological $\mathrm{pH}$ is a highly soluble in fat so fast penetration into the central nervous system, ionization stable $e^{7,10,11}$. Wermeling found that IN midazolam can be absorbed quickly, high bioavailability ${ }^{12}$. Haschke study of intravenous administration of midazolam compared with intranasal showed good pharmacokinetic, absorbed quickly, averaging $72-92 \%$ bioavailability with minimal side effects ${ }^{10}$.

The aim of this study was to find out the efficacy and safety of intranasal (IN) midazolam compared with rectal diazepam in pediatric acute onset seizure.

\section{Material and Methods}

A randomized controlled clinical design experiment was conducted from October 2012 to January 2013. The research was set at the Emergency department (ED) and paediatric inpatient ward at Dr. Soetomo Hospital.

Children with acute all type of seizures admitted to the emergency department and inpatient pediatric ward with one month till 16 years of age at Dr. Soetomo Hospital were enrolled in the study. The patients were excluded if they had respiratory depression, history of allergy to benzodiazepines, status epilepticus and history of acute diarrhea. If seizures did not cease for five minutes after IN midazolam or rectal diazepam administration, it was categorized as treatment failure criteria. The parents obtained informed consent to follow the study. The randomization series list was produced using a computerized random number system. Participants were allocated to one of the two treatment groups using an equal allocation ratio. The allocation sequence was obscured from the investigators enrolling the patients in sequentially numbered and sealed envelopes. The envelopes were then opened after the participants completed all baseline assessments, and that was time to allocate kind of intervention. The randomized codes were kept secure until all data entry was complete. After randomization, patients were randomly assigned to receive intranasal midazolam or rectal diazepam. The patients received IN midazolam at dose $0.2 \mathrm{mg} /$ kgBW into the anterior nares using a mucosal atomizer device (MAD). The Mucosal Atomization Device was an applicator placed on the syringe hub that distributed liquid for nasal administration in a $30-\mu$ particle size, coating the mucosa. It would enhance rapid nasal absorption, reach effective plasma and cerebrospinal fluid concentrations. The other intervention was rectal diazepam at doses of $0.5 \mathrm{mg} / \mathrm{kgBW}$ administrated into the restoration position, lying on their side and gently insert the nozzle of the applicator into the back then pointing it downwards.

Time measurement was immediately performed by using a stopwatch (seconds) when anticonvulsants had been given until the seizures ceased. The patients were observed for one hour after seizures. The seizure was categorized by clinical observation of the physician. The effectiveness of the drug was defined by observation of the cessation of the convulsive activity within five minutes. A history of previous convulsions and antiepileptic medication were obtained from family members.

All data were analyzed in a parametrical statistic. Normality data was tested using Kolmogorov Smirnov test. Median time comparison of seizure cessation between groups was analyzed using log-rank test. Statistical analysis was supported by SPSS 17 for Windows. A $p$ value of $<0.05$ was considered statistically significant.

The ethical clearance certificate had been issued and tested by the Committee on Human Health Research and Ethics Dr. Soetomo Hospital.

\section{Results}

There were 64 subjects appropriate with the study criteria. Four subjects were excluded because of status epilepticus and diarrhea. The baseline characteristics of the subjects before receiving treatment such as variables of age, sex, nutritional status, history of previous seizures, type of seizures were presented in Table 1 . The youngest age was two months, and the oldest was 13 years. The baseline characteristics are homogeneous between the two groups.

Ten subjects had time to seizure cessation more than five minutes, and then they received other anticonvulsants and time to seizure cessation was counted as a sensor that was 300 seconds. Median of time to seizure cessation for IN midazolam group was 42 (12.29 - 54.88) seconds and for rectal diazepam group was 180 (72.8 - 287.1) seconds with significance $p<0.001$. There was statistically significant difference time to seizure cessation between two groups. IN midazolam had a shorter time to stop seizures than rectal diazepam. 
Table 1: Baseline Characteristic of Subjects

\begin{tabular}{|c|c|c|c|}
\hline Characteristic & IN Midazolam n (\%) & Rectal Diazepam n (\%) & p-value \\
\hline \multicolumn{4}{|l|}{ Sex* } \\
\hline Male & $20(66.7)$ & $15(50)$ & 0.295 \\
\hline Female & $10(33.3)$ & $15(50)$ & \\
\hline \multicolumn{4}{|l|}{ Age $($ Year)* } \\
\hline$<1$ & $10(33.3)$ & $25(83.3)$ & 0.722 \\
\hline $1-5$ & $19(63.3)$ & (10) & \\
\hline$>5$ & $1(3.3)$ & $2(6.7)$ & \\
\hline \multicolumn{4}{|l|}{ Nutritional status* } \\
\hline Severe malnourished & $3(10)$ & 0 & \\
\hline Moderate malnourished & $4(13.3)$ & (6.7) & \\
\hline Well nourished & $23(76.7)$ & $26(86.7)$ & 0.148 \\
\hline Overweight & 0 & (3.3) & \\
\hline Obesity & 0 & $1(3.3)$ & \\
\hline \multicolumn{4}{|l|}{ History of seizure ${ }^{\#}$} \\
\hline Yes & $14(46.7)$ & $12(40)$ & 0.794 \\
\hline No & $16(53.3)$ & $18(60)$ & \\
\hline \multicolumn{4}{|l|}{ Type of seizure ${ }^{\#}$} \\
\hline General & $23(76.7)$ & $80)$ & 1 \\
\hline Focal & $7(23.3)$ & $6(20)$ & \\
\hline
\end{tabular}

Significant on $p<0,05 \cdot$ Mann-whitney test, \# Chi square test

Table 2: Time to seizure cessation

\begin{tabular}{|l|c|c|c|}
\hline Variable & IN Midazolam $(\mathbf{n}=30)$ & Rectal Diazepam $(\mathbf{n}=30)$ & $\mathbf{p}$-value \\
\hline Time to seizure cessation (Median in seconds) & $42(29.12-54.88)$ & $180(72.8-287.1)$ & $<0.001$ \\
\hline
\end{tabular}

Significant on $p<0.05$

This study also evaluated the tolerability of IN midazolam, and rectal diazepam administration observed within 24 hours after initiation. It was observed no significant difference in side effects incidence and complications between two groups. Hypoxia occurred in one subject who received IN midazolam, and in two subjects who received rectal diazepam, and they had an improvement in oxygen delivery.

\section{Discussion}

During acute convulsions, the purpose is the immediate termination of the convulsion without physical harming the patient. The proven first choice of drug to be administration is benzodiazepine intravenously. It is difficult to establish intravenous access during the seizure, so noninvasive methods like rectal diazepam are preferred ${ }^{13}$. Some author observed that the parents did not prefer rectal administration ${ }^{3}$. There are physical and social constraints to the use of the rectal route. Rectal diazepam works slower in the onset of action and other disadvantages include the lower social acceptability. When all of these are considered, nasal administration is a simple way without any complication, and the nasopharyngeal mucosa surface is moderately large and well vascularized, tolerating for a rapid absorption of midazolam ${ }^{14}$. It is easier to use midazolam in the nasal drop and spray forms, but these are not available in our country. The only commercially available, undiluted parenteral fluid containing $5 \mathrm{mg} / \mathrm{ml}$ midazolam in Indonesia has been used for this study. This intravenous midazolam formed was administered for a nasal route for acute childhood seizures.

The recent study proved that there was a significant difference in seizure cessation time between IN midazolam group and rectal diazepam on the log rank test with significance $p<0.001$. The mean time required by IN midazolam group to cease seizures was 72.5 seconds and the mean time for rectal diazepam group was 176.9 seconds, with median time for IN midazolam group was 42 (12.29 - 54.88) seconds and for rectal diazepam group was 180 (72.8 - 287.1) seconds. Both the mean and median values in 
the IN midazolam group was smaller than the control group. IN midazolam tends to work faster than rectal diazepam to stop seizures. Midazolam and diazepam are both benzodiazepines group with a similar onset of action, but the determination of the pathways will affect the bioavailability of midazolam through the drug provision ${ }^{10}$. Study from Fisgin et al reported that intranasal midazolam is easy and effective for acute childhood seizures. The study used 16 children at the age of two months to 14 years with a diagnosis of acute seizures. The seizures of three patients terminated within one minute, of seven patients in one to two minutes and three patients in two to five minutes. However, three patients did not respond to treatment ${ }^{15}$. Another research by Bhattacharyya reported the efficacy of intranasal midazolam compared to rectal diazepam. The study used 188 seizure episodes in 46 children, three months to 12 years of age, which randomly assigned. Mean time from arrival of a medical practitioner to drug administration was $68.3 \pm$ 55.12 seconds in diazepam group and $50.6 \pm 14.1$ in seconds in the midazolam group. Mean time from drug administration to a cessation of seizure was significantly less in the midazolam group than the diazepam group ${ }^{16}$. Lahat et al., tried to randomize 52 children with prolonged seizure to receive intravenous diazepam or intranasal midazolam. Good control was achieved equally in both groups, but the mean time from patient arrival to seizure termination was significantly shorter in the patients receiving midazolam. The authors assumed the more rapid effect in the nasal group was determinable to the time saved by excluding the need to insert an intravenous line ${ }^{17}$.

Intranasal midazolam was also found to be a good option for home administration by parents. Wilson et al., stated that 33 of 40 parents questioned reported intranasal midazolam to be effective, and $83 \%$ preferred it to rectal diazepam ${ }^{18}$.

The nasal mucosa provides a large, highly vascular absorptive surface adjacent to the brain. Together with neighboring olfactory mucosa, it offers a direct pathway for drug absorption into the bloodstream and cerebrospinal fluid. At physiological $\mathrm{PH}$, the ring formed by the molecule closes, making it highly lipophilic. Then, as a result, it overpasses the blood-brain barrier and flows into the central nervous system. Therefore, the nasal route is a good possibility for medication that undergo massive first-pass hepatic metabolism, and drugs with erratic absorption pattern ${ }^{19}$. When intranasal midazolam is administered, it is available in the cerebral cortex in 2 to 5 minutes, and beta activity increases in the electroencephalogram (EEG). It takes a maximum of eight minutes to achieve a positive response ${ }^{20}$.

The dose of intranasal midazolam for terminating seizure is based on body weight. According to Knoester et al., the dose recommended is $0.2 \mathrm{mg} / \mathrm{kg}$ for children ${ }^{14}$. This study used the similar dose appropriate for those previous study. When engaging the intranasal route for benzodiazepines, it is essential that the drug be highly intensified and that it be distributed directly to the surface of the mucosa. Too abundant an amount or over rapid administration may result in suboptimal absorption and loss of drug into the pharynx, rendering it ineffective ${ }^{19}$.

The intranasal route appears to be equally safe to intravenous and intramuscular routes, which have not been observed to be associated with respiratory changes. Tolerability that was assessed in this study is the appearance of the side effects of the administration of benzodiazepines. There were three subjects who suffer from hypoxia with oxygen saturation was below $80 \%$. Moreover, there was a subject with pneumonia whose saturation was $70 \%$. In this case, probably hypoxia was not caused by medication given but from the underlying disease of the subject. Two other subjects with a diagnosis of cerebral abscess, oxygen saturation improved after the therapy was given, in this situation hypoxia, appeared due to benzodiazepine administration, which was immediately improved after administration of oxygen. There were no subjects who experienced any side effects such as respiratory depression, hypotension, cough and allergic reactions. Lahat et al., administered intranasal midazolam to 20 children aged six months to five years with acute seizures. In 19 children, control was accomplished within 3.5 minutes of drug distribution, and none of the children had clinical symptoms of respiratory distress or bradycardia ${ }^{21}$. These findings have important implications, as the specific emergency treatment used may be part of the many issues responsible for respiratory depression ${ }^{19}$. In spite of the existence of an upper respiratory tract infection might help drug absorption by enhancing blood flow to the nasal mucosa, nasal secretions can dilute the midazolam solution and interfere with its contact with the absorbing surface. Lahat et al., reported most of the 21 children followed the instruction had an upper respiratory tract infection; otherwise only three children revealed ineffective absorption of midazolam and subsequent poor seizure termination ${ }^{17}$. Lugo et al., reported pain and irritation during nasal administration ${ }^{22}$. However, we did not observe that kind of side effects.

This study limits to small sample size due to difficulties of finding seizing children in emergency 
room and pediatric ward. Although there was seizing child, the evident just only took a few seconds and ended itself. The seizure type was also unclassified because of a technical problem of testifying the incident.

\section{Conclusion}

In conclusion, IN midazolam was found to be more effective than rectal diazepam to terminate acute seizures in children. Its anticonvulsant route effect is reasonably safe and easy to be administered. This study is indicating that intranasal midazolam may be used not only in medical centers but also in general practitioner's office as well as at home by parents and families of seizure-prone children, after appropriate instruction. Future studies with electroencephalographic recording are recommended to ensure the efficacy of intranasal midazolam as an alternative route in the treatment of pediatric seizures.

Acknowledgements: We are thankful for M Riza Kurniawan for helping data collection

Funding: Nil

Conflicts of interest: Nil

Permission from IRB: Yes

\section{References}

1. McAbee GN, Wark JE. A practical approach to uncomplicated seizures in children. Am Fam Physician 2000;62(5):1109-16.

2. Friedman MJ, Sharieff GQ. Seizures in children. Pediatr Clin N Am 2006; 53:257-77. doi:10.1016/j. pcl.2005.09.010

3. Fisgin T, Gurer Y, Tezic T, Senbil N, Zorlu P, Okuyas P.Effect of intranasal midazolam and rectal diazepam on acute convulsions in children: prospective randomized study. J Child Neurol 2002;17:123-6

4. Doshi D. Controlling seizures in children: diazepam or midazolam ? systematic review. Hong Kong J Emerg Med 2010; 17:196-204

5. Vilke G, Sharieff GQ, Marino A, Gerhart AE, Chan TC. Midazolam for treatment of out of hospital pediatric seizure. Prehospital Emerg care 2002; 6:215-7

6. Shah I, Deshmukh CT. Intramuscular midazolam vs intravena diazepam for acute seizures. Indian J Pediatr 2005;72:667-70

7. Paun JS, Bagada AA, Raval MK. Nasal drug deliveryAs an effective tool for brain targeting-A review. IJPAS 2010;1:43-53
8. S. Botner and A. Sintov, "Intranasal Delivery of Two Benzodiazepines, Midazolam and Diazepam, by a Microemulsion System," Pharmacology \& Pharmacy, 2011;2(3):180-88. doi: 10.4236/ pp.2011.23026.

9. Wermeling DP. Intranasal Delivery of Antiepileptic Medications for Treatment of Seizures. Neurotherapeutics 2009;6:352-58. doi: 10.1016/j. nurt.2009.01.002

10. Haschke M.Suter K, Hofmann S, Witschi R, Frohlich J, Imandinis $\mathrm{G}$ et al. Pharmacokinetics and pharmacodynamics of nasally delivered midazolam. Br J Clin Pharmacol 2010;69:607-16. doi: 10.1111/j.1365-2125.2010.03611.x.

11. Holsti M, Dudley N, Schunk J, Adelgais K, Greenberg R, Olsen C, Healy A, Firth S, Filloux F. Intranasal midazolam vs rectal diazepam for the home treatment of acute seizures in pediatric patients with epilepsy. Arch Pediatr Adolesc Med 2010;164:747-53. doi: 10.1001/ archpediatrics.2010.130

12. Wermeling DP, Record K, Kelly T, Archer S, Clinch T, Rudy A. Pharmacokinetics and pharmacodynamics study of a new intranasal midazolam formulation in healthy volunteers. Anesth Analg 2006;103:34453

13. Lejus C, Renaudin M, Testa S, Malinovsky JM, Vigier T, Souron R. Midazolam for premedication in children: nasal vs. rectal administration. Eur J Anaesthesiol 1997;14:244-49.

14. Knoester PD, Jonker DM, Hoeven RTM, Vermeji TAC, Edelbroek PM, Brekelmans GJ. Pharmacokinetics and pharmacodynamics of midazolam administered as a concentrated intranasal spray. A study in healthy volunteers. $\mathrm{Br}$ J Clin Pharmacol 2002;53:501-7.

15. Fisgin $T$, Gurer $Y$, Senbil N, Tezic $T$, Zorlu $P$, Okuyaz $C$, et al. Nasal midazolam effects on childhood acute seizures. J Child Neurol 2000;15:833-5.

16. Bhattacharyya M, Kalra V, Gulati S. Intranasal Midazolam vs Rectal Diazepam in Acute Childhood Seizures. Pediatr Neurol 2006;34:355-59

17. Lahat E, Goldman M, Barr J, Bisritzer T, Berkovitch M. Comparison of intranasal midazolam with intravenous diazepam for treating febrile seizures in children: prospective randomized study. BMJ 2000;321(7253):83-6.

18. Wilson MT, Macleod S, O’Regan ME. Nasal/buccal midazolam use in community. Arch Dis Child 2004;89:50-51. 
19. Kremer AK, Goldman RD. Intranasal midazolam for treatment of seizures in children in the emergency setting. Israel J Emerg Med 2007;7:31-5.

20. Niall CT, Leigh J, Rosen DR, Pandit UA. Preanesthetic sedation of preschool children using intranasal midazolam. Anesthesiology 1998;69:972-5.
21. Lahat E, Goldman M, Barr J, Eshel G, Berkovitch M. Intranasal midazolam for childhood seizures. Lancet 1998;352(9128):620.

22. Lugo RA, Fishbein $M$, Nahata $M C$, Lininger $B$. Complication of intranasal midazolam. Pediatrics 1993;92:638. 